\title{
A novel approach to noninvasive measurement of overhead line impedance parameters
}

Article

Accepted Version

Ritzmann, D., Rens, J., Wright, P. S., Holderbaum, W. and Potter, B. (2017) A novel approach to noninvasive measurement of overhead line impedance parameters. IEEE Transactions on Instrumentation and Measurement, 66 (6). pp. 1155-1163. ISSN 0018-9456 doi:

https://doi.org/10.1109/TIM.2017.2665958 Available at https://centaur.reading.ac.uk/69898/

It is advisable to refer to the publisher's version if you intend to cite from the work. See Guidance on citing.

Published version at: http://ieeexplore.ieee.org/document/7865895/

To link to this article DOI: http://dx.doi.org/10.1109/TIM.2017.2665958

Publisher: IEEE

All outputs in CentAUR are protected by Intellectual Property Rights law, including copyright law. Copyright and IPR is retained by the creators or other copyright holders. Terms and conditions for use of this material are defined in the End User Agreement. 


\section{CentAUR}

Central Archive at the University of Reading

Reading's research outputs online 


\title{
A Novel Approach to Non-invasive Measurement of Overhead Line Impedance Parameters
}

\author{
Deborah Ritzmann, Student Member, IEEE, Johan Rens, Member, IEEE, Paul S. Wright, \\ William Holderbaum, Member, IEEE, and Ben Potter, Member, IEEE
}

\begin{abstract}
Transmission line impedance parameter estimation forms an essential part in modelling and monitoring of electricity networks. The accuracy of the estimated parameters has a direct impact on various functions of network operation, such as state estimation, fault location and dynamic thermal line rating. Impedance parameters can be estimated from voltage and current measurements taken at the ends of the transmission line. Previous research has produced a range of methods with the purpose of maximizing the accuracy of the estimated values. However, the input measurements from the line ends can contain systematic errors that are introduced by the instrumentation channel and significantly reduce impedance parameter accuracy, which is not taken into account by most of the existing methods. In this paper, a novel method is presented that estimates correction factors for the systematic errors and thus increases the accuracy of impedance parameter values. The performance of the new method is compared to an existing one in a case study on laboratory measurements.
\end{abstract}

Index Terms-Accuracy, admittance measurement, impedance measurement, optimization methods, parameter estimation, phasor measurement unit (PMU), power transmission, transmission line measurements.

\section{INTRODUCTION}

A CROSS the world, more and more households and businesses are relying on stable provision of electricity in their daily activities. At the same time, there is a drive to increase the amount of renewable energy generation to mitigate the effects of climate change. In order to meet both demands, existing and new applications for safe and optimal operation of electricity networks are being developed. Many of these applications are underpinned by coherent network models and data. The impedance parameters of overhead transmission lines form an essential part in electricity network modelling, and their accuracy has an impact on the efficiency of network operation; examples include state estimation [?], fault location [?] and protection [?]. For this reason, there is an incentive to continually improve the accuracy of overhead line impedance parameter estimation.

Impedance parameters can be calculated by computer programs that are based on electromagnetic theory and take into

This work was supported by the Climate Knowledge and Innovation Community (Climate-KIC) within the European Institute of Innovation and Technology (EIT).

D. Ritzmann, W. Holderbaum and B. Potter are with the University of Reading, Reading, RG6 6UR, UK (e-mail: d.ritzmann@pgr.reading.ac.uk; w.holderbaum@reading.ac.uk; b.a.potter@reading.ac.uk).

P. S. Wright is with the National Physical Laboratory, Teddington, TW11 OLW, UK (e-mail: paul.wright@npl.co.uk).

J. Rens is with the North-West University, Private Bag X6001, Potchefstroom, 2520, SA, (e-mail: johan.rens@nwu.ac.za). account the electric properties and geometrical configuration of the conductors [?]. Alternatively, it is possible to measure impedance parameters indirectly, with measurements of signals at the line ends as input quantities to a measurement model for the overhead line system. The advantage of indirect measurement of impedance parameters is that longterm changes due to aging of the conductors and short-term changes due to temperature variation can be tracked if there is a continuous stream of measurements from the energized line.

The accuracy of the input measurements determines the accuracy of the estimated impedance parameter values. A previous paper by the authors proposed to investigate the effect of off-nominal frequencies and harmonics on impedance parameter estimation accuracy [?] and many of the existing methods consist of a linear or non-linear estimator that filters random noise from the input measurements [?], [?], [?], [?], [?], [?]. In addition, statistical methods for the detection of individual bad measurements can be implemented [?].

Most of these methods do not consider the fact that both SCADA (Supervisory Control And Data Acquisition) and synchrophasor measurements can be subject to significant systematic errors that arise in the substation instrumentation channel due to instrument transformers, cables and burdens [?]. Instrumentation channels are rarely calibrated due to the associated line outages and costs. Various approaches have been proposed for synchrophasor measurement based calibration [?], [?]; but to the best of the authors' knowledge, these assume that accurate transmission line impedance parameter values are already available. For this reason, simultaneous estimation of calibration factors and impedance parameters has been suggested [?], [?]. If impedance parameters and calibration factors for both voltage and current are to be found, the problem has a high number of unknowns and is prone to becoming ill-conditioned, even if it is over-determined.

A different approach to estimate correction factors for current and voltage measurements exists [?]. Instead of estimating specific impedance parameter values, assumptions are made about the variation of the parameter values over time, based on expected physical behaviour of overhead line conductors. However, this approach identified correction factors for measurements at one line end only, which limits the achievable impedance parameter accuracy. Moreover, the method assumed that any set of input measurements results in useful correction factors. In order to resolve these issues, this paper proposes a novel method that builds on the existing approach. The new method also relies on assumptions about the expected physical behaviour of overhead lines. 


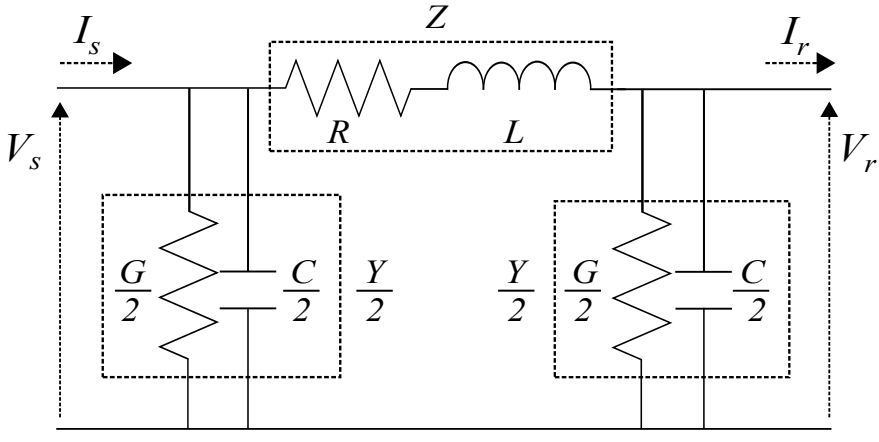

Fig. 1. Equivalent pi-circuit for a transmission line.

Correction factors for systematic errors in measurements at both line ends are estimated; furthermore, the method finds correction factors from a range of available input measurement sets and uses measurements of average line temperature to select those correction factors that give the best impedance parameter estimates.

The rest of the paper has the following structure: Section II outlines the new solution approach and briefly describes an existing method for comparison. In Section III, results from a case study on laboratory measurements are presented. Section IV discusses practical implementation as well as limits and areas for future work. Section V concludes the paper.

\section{METHODS}

\section{A. A new method for impedance parameter estimation}

1) Description of the measurement model: The symmetric pi-circuit as shown in Fig. 1 consists of three lumped components: the series impedance $Z$, and two identical shunt admittances $Y / 2$, where

- $Z=R+j X, Y=G+j B$

- $X=2 \pi f L, B=2 \pi f C$

- $Z, Y \in \mathbb{C}, R, L, X, G, C, B, f \in \mathbb{R}$

- $R$ is the resistance, $L$ is the inductance, $X$ is the reactance, $G$ is conductance, $C$ is the capacitance and $B$ is the susceptance, $f$ is the frequency.

The lumped components are equivalent to the distributed parameters of a long transmission lines [?].

Let the synchronous measurements of voltage and current at time instant $t_{i}, i=1,2,3, \ldots, n$ be $V_{s_{i}}, I_{s_{i}}, V_{r_{i}}, I_{r_{i}} \in \mathbb{C}$; subscripts $s$ and $r$ refer to sending and receiving ends, respectively. Further, let $a_{s}, a_{r}, b_{s}, b_{r} \in \mathbb{C}$ be the correction factors that compensate for systematic errors in the synchrophasor measurements. Expressions for impedance $Z_{i}=R_{i}+j X_{i}$ and admittance $Y_{i}=G_{i}+j B_{i}$ at $t_{i}$ are obtained from Kirchhoff's laws:

$$
\begin{aligned}
Z_{i} & =\frac{\left(a_{s} V_{s_{i}}\right)^{2}-\left(a_{r} V_{r_{i}}\right)^{2}}{\left(a_{s} V_{s_{i}}\right)\left(b_{r} I_{r_{i}}\right)+\left(a_{r} V_{r_{i}}\right)\left(b_{s} I_{s_{i}}\right)} \\
Y_{i} & =2 \frac{b_{s} I_{s_{i}}-b_{r} I_{r_{i}}}{a_{s} V_{s_{i}}+a_{r} V_{r_{i}}} .
\end{aligned}
$$

The circuit model and equations are for a single-phase transmission line, which is equivalent to a three-phase system if the line has perfect phase symmetry. Phase symmetry is a reasonable assumption for transposed transmission lines. In steady-state, balanced operation, the positive sequence of the fundamental frequency component of voltage and current closely represents the line's state and is thus of interest for many power system management applications. Hence, in this paper $V_{s_{i}}, I_{s_{i}}, V_{r_{i}}, I_{r_{i}}, Z_{i}, Y_{i}$ are assumed to be positive sequence quantities at the fundamental system frequency.

The parameter estimation problem consists of determining values for $a_{s}, a_{r}, b_{s}, b_{r}$ from measurements $V_{s_{i}}, I_{s_{i}}, V_{r_{i}}, I_{r_{i}}$ so that $Z_{i}$ and $Y_{i}$ can be calculated accurately.

2) Identification of correction factors for systematic errors: The proposed method builds on an existing algorithm [?], whereby correction factors are determined from $n$ sets of consecutive synchrophasor measurements by solving an optimization problem.

An underlying assumption of this method is that over the time period of the $n$ measurements the conductor electrical properties follow a certain behaviour. Inductance $L$ and capacitance $C$ can be affected by temperature variations because of changes in the conductor length [?]. But the variation is of the order of $0.001 \%$, which is smaller than achievable uncertainties $(0.1 \%$ [?]). Thus, $L$ and $C$ are assumed constant over time. Conductance $G$ depends on the surface of the conductor and it can vary with changing levels of humidity, but because its overall magnitude is normally less than $10 \mu \mathrm{S}$ [?], it can also be assumed constant. Bare overhead line conductors have resistance-temperature coefficients of the order of $0.1 \%$, which means that resistance $R$ varies significantly with line current and ambient conditions [?], [?]. Therefore it is assumed that during the period $t_{n}-t_{1}$ resistance $R$ varies linearly. To describe these physical behaviours, define model functions $f_{R}, f_{X}, f_{B}: \mathbb{R}_{\geq 0} \rightarrow \mathbb{R}$ for $R, X, B$, respectively, where

$$
f_{R}\left(t_{i}\right)=q_{R} t_{i}+r_{R}
$$

and $q_{R}, r_{R} \in \mathbb{R}$ are model parameters, which are computed through a linear least squares fit of the $n$ parameter values $R_{i}$, calculated at time instants $t_{i}, i=1,2,3, \ldots, n$. Details of estimation of $q_{R}, r_{R}$ are referred to previous work [?]. $f_{X}, f_{B}$ are constant functions, whose values $r_{X}, r_{B} \in \mathbb{R}$ are the mean parameter values over time period $t_{n}-t_{1}$ :

$$
f_{X}\left(t_{i}\right)=r_{X}=\frac{1}{n} \sum_{i=1}^{n} X_{i} ; f_{B}\left(t_{i}\right)=r_{B}=\frac{1}{n} \sum_{i=1}^{n} B_{i} .
$$

Systematic errors in the voltage and current measurements cause the calculated values $R_{i}, X_{i}, B_{i}$ to deviate from their physically expected, linear behaviour with respect to time, that means a deterioration in the goodness of fit of $f_{R}, f_{X}, f_{B}$ to $R_{i}, X_{i}, B_{i}$, respectively. Similarly, the calculated values $G_{i}$ become unphysical $\left(G_{i}>>10 \mu \mathrm{S}\right)$. As a measure for the goodness of fit, the sum of the squares of the residuals of the linear least squares fit of (3) is defined as

$$
S_{R}=\sum_{i=1}^{n}\left(R_{i}-f_{R}\left(t_{i}\right)\right)^{2}
$$

where $S_{R} \in \mathbb{R}_{\geq 0} . S_{X}$ and $S_{B}$ are defined similarly in terms of $X_{i}, f_{x}$ and $\bar{B}_{i}, f_{B}$, respectively. 
The effect of systematic errors on $G_{i}$ is measured by $S_{G} \in$ $\mathbb{R}_{\geq 0}$ where

$$
S_{G}=\sum_{i=1}^{n} G_{i}^{2}
$$

The objective is to find values for correction factors $a_{s}, a_{r}, b_{s}, b_{r}$ that maximise the goodness of fit, which is achieved by minimizing $S_{R}, S_{X}, S_{G}, S_{B}$. Hence, the following optimization problem is defined: let $h \in \mathbb{R}^{8}$,

$$
\begin{array}{cl}
h=\left(\left|a_{s}\right|, \arg \left(a_{s}\right),\left|a_{r}\right|, \arg \left(a_{r}\right),\left|b_{s}\right|, \arg \left(b_{s}\right),\left|b_{r}\right|, \arg \left(b_{r}\right)\right), \\
\underset{h}{\operatorname{minimize}} & g(h)=\mu_{R}^{2} S_{R}+\mu_{X}^{2} S_{X}+\mu_{G}^{2} S_{G}+\mu_{B}^{2} S_{B} \\
\text { subject to } & \left|a_{s}\right|,\left|\arg \left(a_{s}\right)\right|,\left|a_{r}\right|,\left|\arg \left(a_{r}\right)\right|<0.1, \\
& \left|b_{s}\right|,\left|\arg \left(b_{s}\right)\right|,\left|b_{r}\right|,\left|\arg \left(b_{r}\right)\right|<0.1
\end{array}
$$

with initial values $a_{s}=a_{r}=b_{s}=b_{r}=1$.

$S_{R}, S_{X}, S_{G}, S_{B}$ are functions of $h$ since $R_{i}, X_{i}, G_{i}, B_{i}$ are calculated using $a_{s}, a_{r}, b_{s}, b_{r}$. The factors $\mu_{R}, \mu_{X}, \mu_{G}, \mu_{B} \in$ $\mathbb{R}_{\geq 0}$ are weighting factors that can be used to adjust the relative magnitudes of the terms of $g(h)$. The choice of weighting factors has an effect on the optimized goodness of fit of $f_{R}, f_{X}, f_{B}$ to $R_{i}, X_{i}, B_{i}$ and the overall magnitude of $G_{i}$, that means to what extent the identified correction factors make the calculated impedance and admittance more compliant to expected physical behaviour. For instance, if $\mu_{R}=\mu_{X}=\mu_{G}=\mu_{B}=1, S_{R}$ and $S_{X}$ will dominate and no significant improvement may occur in $S_{G}, S_{B}$.

The inequality constraints arise from existing accuracy classes of instrument transformers [?], [?] and characterization of instrumentation channels [?], which imply that systematic errors in voltage and current magnitude do typically not exceed $\pm 10 \%$, and errors in phase angle are less than $\pm 0.1 \mathrm{rad}$.

Equation (7) is a non-linear, constrained optimization problem. The objective function $g(h): \mathbb{R}^{8} \rightarrow \mathbb{R}_{\geq 0}$ is a linear combination of the four independent variables $S_{R}, S_{X}, S_{G}, S_{B}$ with positive constants $\mu_{R}, \mu_{X}, \mu_{G}, \mu_{B}$. Therefore $g(h)$ is a convex function and any local minimum of $g(h)$ is a global minimum. Various algorithms are available that can efficiently identify local minima for non-linear, constrained optimization problems. In this paper, the interior-point method is used [?].

3) Selection of optimal correction factors: A set of $n$ synchrophasor measurements of voltage and current is required to obtain correction factors $a_{s}, a_{r}, b_{s}, b_{r}$. There remains a question about how large $n$ should be and which measurements should be chosen. For this reason, it is proposed to obtain correction factors from a range of measurement subsets.

Let $\Lambda \in \mathbb{N}$ be the number of subsets selected according to steps detailed in Appendix A. Correction factors $a_{s_{\lambda}}, a_{r_{\lambda}}, b_{s_{\lambda}}, b_{r_{\lambda}}, \lambda=1, \ldots, \Lambda$ are obtained from each subset using the proposed method. The correction factors are used to calculate impedance parameter estimates $\hat{Z}_{i_{\lambda}}$ and $\hat{Y}_{\lambda}$, details of which are given in Appendix B. To assess the accuracy of impedance parameter values, residuals $S_{V_{\lambda}}, S_{I_{\lambda}}$ of calculated receiving end voltage and current $\hat{V}_{r_{i}}, \hat{I}_{r_{i}}$ are evaluated. $\hat{V}_{r_{i}}, \hat{I}_{r_{i}}$ are calculated by rearranging (1) and (2):

$$
\begin{aligned}
& \hat{V}_{r_{i}}=\left(1+\hat{Y}_{\lambda} \hat{Z}_{i_{\lambda}} / 2\right)\left(a_{s_{\lambda}} V_{s_{i}}\right)-\hat{Z}_{\lambda}\left(b_{s_{\lambda}} I_{s_{i}}\right) \\
& \hat{I}_{r_{i}}=\left(1+\hat{Y}_{\lambda} \hat{Z}_{i_{\lambda}} / 2\right)\left(b_{s_{\lambda}} I_{s_{i}}\right)-\left(\hat{Y}_{\lambda}+\hat{Y}_{\lambda}^{2} \hat{Z}_{i_{\lambda}}\right)\left(a_{s_{\lambda}} V_{s_{i}}\right) .
\end{aligned}
$$

$S_{V_{\lambda}}, S_{I_{\lambda}} \in \mathbb{R}_{>0}$ are the root-mean-square of the voltage and current residuals, respectively, given by:

$$
\begin{aligned}
& S_{V_{\lambda}}=\sqrt{\frac{1}{N} \sum_{i=1}^{N}\left|\hat{V}_{r_{i}}-a_{r_{\lambda}} V_{r_{i}}\right|^{2}} \\
& S_{I_{\lambda}}=\sqrt{\frac{1}{N} \sum_{i=1}^{N}\left|\hat{I}_{r_{i}}-b_{r_{\lambda}} I_{r_{i}}\right|^{2}}
\end{aligned}
$$

In addition, the root-mean-square of the temperature residual $S_{T_{\lambda}}$ is evaluated using temperature estimates $\hat{T}_{c_{i}}$ and temperature measurements $T_{c_{i}}$. Details of the estimation of $\hat{T}_{c_{i}}$ are given in Appendix B. $S_{T_{\lambda}} \in \mathbb{R}_{\geq 0}$ is given by

$$
S_{T_{\lambda}}=\sqrt{\frac{1}{N} \sum_{i=1}^{N}\left[\hat{T}_{c_{i}}-T_{c_{i}}\right]^{2}} .
$$

$S_{V_{\lambda}}, S_{I_{\lambda}}, S_{T_{\lambda}}$ are combined to give a single quantity $S_{V I T_{\lambda}}$ : $\mathbb{R}^{3} \rightarrow \mathbb{R}_{\geq 0}$

$$
S_{V I T_{\lambda}}\left(S_{V_{\lambda}}, S_{I_{\lambda}}, S_{T_{\lambda}}\right)=S_{V_{\lambda}} S_{I_{\lambda}} S_{T_{\lambda}}
$$

Once $S_{V I T_{\lambda}}$ has been evaluated for all $\Lambda$ measurement sets, the optimal set of correction factors is that with the minimum value of $S_{V I T_{\lambda}}$. The selected correction factors can then be used to calculate $Z, Y$ and $T_{c}$ from synchrophasor measurements.

\section{B. Summary of an existing method for impedance parameter estimation}

For comparison, a summary of an existing method for synchrophasor-based impedance parameter and temperature estimation is given in this section. The existing method aims to maximize parameter accuracy by estimating values for both the impedance parameters as well as the synchrophasor measurements using non-linear estimation theory [?]. Resistance is assumed to be constant over the measurement period. For simplicity, equivalent instead of distributed line parameters are used in this paper. The objective function is based on Kirchhoff's voltage law for the pi-circuit:

$$
V_{s}-I_{s} Z+V_{s} Z Y / 2-V_{r} \exp (j \beta)=0,
$$

where $\beta \in[-\pi, \pi]$ is a synchronization angle, which allows for detection and correction of a synchronization error between sending and receiving end measurements. Since the receiving end current $I_{r}$ does not occur in (14), $\beta$ corrects only the phase angle of $V_{r}$.

Suppose that $n$ sets of synchrophasor measurements $\left\{V_{s_{k}}, I_{s_{k}}, V_{r_{k}}\right\}, k=1,2,3, \ldots, n$ are used for parameter estimation. Let

$$
\begin{aligned}
& v_{s_{k}}=\left|V_{s_{k}}\right|, \quad w_{s_{k}}=\left|I_{s_{k}}\right|, \quad v_{r_{k}}=\left|V_{r_{k}}\right|, \\
& \theta_{s_{k}}=\arg \left(V_{s_{k}}\right), \rho_{s_{k}}=\arg \left(I_{s_{k}}\right), \theta_{r_{k}}=\arg \left(V_{r_{k}}\right)
\end{aligned}
$$

and define the vector of unknowns $\mathbf{P} \in \mathbb{R}^{5+6 n}, \mathbf{P}=$ $\left[R, X, G, B, \beta, v_{s_{k}}, w_{s_{k}}, v_{r_{k}}, \theta_{s_{k}}, \rho_{s_{k}}, \theta_{r_{k}}\right]$. Define a vector of objective functions $\mathbf{F}(\mathbf{P}) \in \mathbb{R}^{8 n+1}$, 
$\mathbf{F}=\left[\eta, f_{1_{k}}, f_{2_{k}}, g_{1_{k}}, g_{2_{k}}, g_{3_{k}}, g_{4_{k}}, g_{5_{k}}, g_{6_{k}}\right]^{T}, \quad f_{1_{k}}, f_{2_{k}} \quad:$ $\mathbb{R}^{11} \rightarrow \mathbb{R}, \eta, g_{1_{k}}, g_{2_{k}}, g_{3_{k}}, g_{4_{k}}, g_{5_{k}}, g_{6_{k}}: \mathbb{R} \rightarrow \mathbb{R}$, where

$$
\begin{gathered}
f_{1_{k}}=\operatorname{Re}\left(V_{s_{k}}-I_{s_{k}} Z+V_{s_{k}} Z Y / 2-V_{r_{k}} \exp (j \beta)\right), \\
f_{2_{k}}=\operatorname{Im}\left(V_{s_{k}}-I_{s_{k}} Z+V_{s_{k}} Z Y / 2-V_{r_{k}} \exp (j \beta)\right) \\
\eta=\beta, g_{1_{k}}=v_{s_{k}}, g_{2_{k}}=w_{s_{k}}, g_{3_{k}}=v_{r_{k}} \\
g_{4_{k}}=\theta_{s_{k}}, g_{5_{k}}=\rho_{s_{k}}, g_{6_{k}}=\theta_{r_{k}} .
\end{gathered}
$$

The voltage and current magnitudes $v, w$ and phase angles $\theta, \rho$ as well as synchronization angle $\beta$ are included in the vector of unknowns $\mathbf{P}$ and vector of measurement functions $\mathbf{F}(\mathbf{P})$ such that the residuals between estimated values and measured or assumed values can be used to detect individual measurements with errors larger than the measurement uncertainty; these can be caused by transient failure of the measurement instrumentation.

Furthermore, define the measurement vector $\mathbf{M} \in \mathbb{R}^{8 n+1}$ such that $\mathbf{M}=\left[M_{i}, v_{s_{k}}, w_{s_{k}}, v_{r_{k}}, \theta_{s_{k}}, \rho_{s_{k}}, \theta_{r_{k}}\right], M_{i}=0, i=$ $1,2,3, \ldots, 2 n+1, k=1,2,3, \ldots, n$. For accurate parameter estimates, it is expected that the function vector $\mathbf{F}(\mathbf{P})$ yields values close to the measurement vector $\mathbf{M}$. Thus it is assumed that

$$
\mathbf{M}=\mathbf{F}(\mathbf{P})+\boldsymbol{\epsilon}
$$

where $\epsilon \in \mathbb{R}^{8 n+1}$ represents the differences between measured and calculated values, which are expected to occur due to measurement uncertainty. By minimizing $\mathbf{J} \in \mathbb{R}^{8 n+1}$,

$$
\mathbf{J}=[\mathbf{M}-\mathbf{F}(\mathbf{P})]^{T}[\mathbf{M}-\mathbf{F}(\mathbf{P})],
$$

an optimal estimate of $\mathbf{P}$ is found. An iterative algorithm is used to obtain a parameter estimate $\hat{\mathbf{P}}=\left[\hat{R}, \hat{X}, \hat{G}, \hat{B}, \hat{\beta}, \hat{v}_{s_{k}}, \hat{w}_{s_{k}}, \hat{v}_{r_{k}}, \hat{\theta}_{s_{k}}, \hat{\rho}_{s_{k}}, \hat{\theta}_{r_{k}}\right]$. The chisquare test is applied to detect bad measurements, which are removed and a new parameter estimate is obtained from the remaining measurements. An estimate of the average line temperature $T_{c}$ can be calculated using the linear resistance-temperature relationship [?]:

$$
\hat{T}_{c}=T_{0}+\frac{1}{\alpha}\left(\hat{R}-R_{0}\right),
$$

where handbook values of reference values $R_{0}$ at $T_{0}$ and resistance-temperature coefficient $\alpha$ are used. In order to monitor variations in line resistance and temperature, $\hat{\mathbf{P}}$ has to be computed at regular time intervals using a set of synchrophasor measurements taken over a short period of time, during which the resistance is assumed constant.

\section{CASE STUDY}

In this section, the existing method (Section II-B) as well as the proposed new method (Section II-A) are applied to estimate the impedance parameters of a specific overhead transmission line. Firstly, details about the line's properties and measurements are given; then the results from a laboratorybased line simulation are presented.
TABLE I

NOMINAL TRANSMISSION LINE PARAMETER VALUES

\begin{tabular}{lcc}
\hline & Per unit length & Equivalent \\
\hline Resistance $R_{0}$ at $T_{c}=20^{\circ} \mathrm{C}$ & $34.5 \mathrm{~m} \Omega \mathrm{km}^{-1}$ & $16.2 \Omega$ \\
Inductance $L$ & $1.21 \mathrm{mH} \mathrm{km}^{-1}$ & $600 \mathrm{mH}$ \\
Reactance $X$ at $f=50 \mathrm{~Hz}$ & $381 \mathrm{~m} \Omega \mathrm{km}^{-1}$ & $189 \Omega$ \\
Capacitance $C$ & $9.35 \mathrm{nF} \mathrm{km}^{-1}$ & $5.00 \mu \mathrm{F}$ \\
Susceptance $B$ at $f=50 \mathrm{~Hz}$ & $2.94 \mu \mathrm{km}^{-1}$ & $1.57 \mathrm{mS}$ \\
\hline
\end{tabular}

\section{A. Transmission line properties and measurements}

The overhead transmission line under consideration is operated at a nominal voltage level of $330 \mathrm{kV}$ and at a nominal frequency of $50 \mathrm{~Hz}$; its length is $521 \mathrm{~km}$ and it is fully transposed. The MATLAB ${ }^{\circledR}$ Simscape $^{\mathrm{TM}}$ Power Systems ${ }^{\mathrm{TM}}$ program power_lineparam has been used to calculate per unit length values of positive sequence resistance at $20^{\circ} \mathrm{C}$ as well as inductance and capacitance of the overhead line, based on the conductor types, tower geometry and electromagnetic theory [?]. The calculated results are listed in Table I, along with values of the equivalent lumped pi-circuit components shown in Fig. 1, which represent the uniformly distributed parameters.

As was noted in Section II-A3, the proposed approach for parameter estimation requires values of the average line temperature. The average conductor temperature was calculated using the heat-balance equation for bare overhead conductors [?]. The calculation was based on measurements of ambient temperature, wind speed and solar radiation, which were based on average values from two weather stations closest to the line ends.

\section{B. Transmission line simulation}

A software simulation of the transmission line was implemented in MATLAB ${ }^{\circledR}$ Simscape $^{\mathrm{TM}}$ Power Systems ${ }^{\mathrm{TM}}$. The transmission line under consideration was modelled as a symmetric, three-phase line with uniformly distributed parameters and length as specified in Section III-A. The assumed variation in positive sequence resistance was calculated using the estimated line temperature and a handbook value of $\alpha=0.0039^{\circ} \mathrm{C}^{-1}$ for the resistance-temperature coefficient [?], [?].

Equivalent voltage sources were used to model the network at either line end; their magnitudes and phase angles were varied to give a range of operating conditions spread over a 24hour period. Fig. 2 and 3 show voltage and current magnitudes, respectively.

\section{Laboratory-based measurements}

Values of voltage and current at the line ends were taken from the software simulation and used as input files for two physical waveform generators. The voltage and current signals were played out simultaneously, then captured by two power quality instruments that report time-tagged phasors at the fundamental frequency. A diagram of the experimental setup is shown in Fig. 4. 


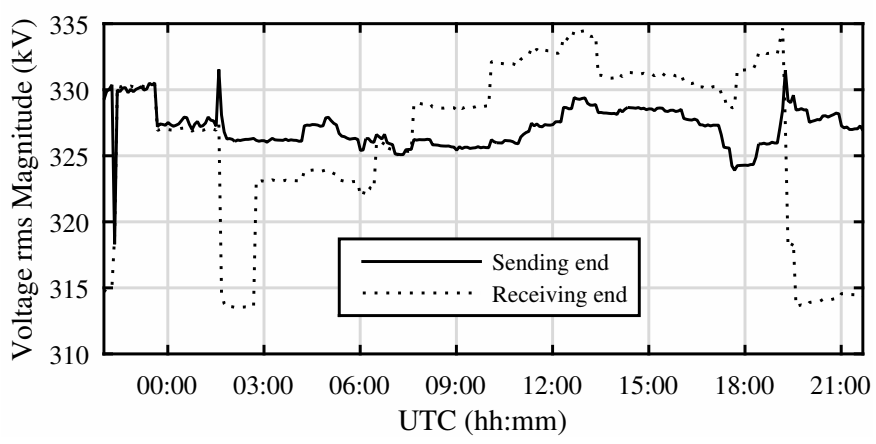

Fig. 2. Magnitude of the sending and receiving end voltages.

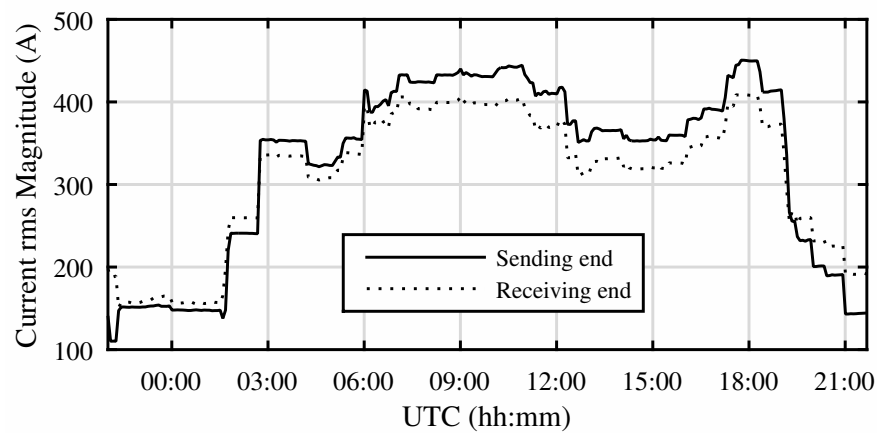

Fig. 3. Magnitude of the sending and receiving end currents.

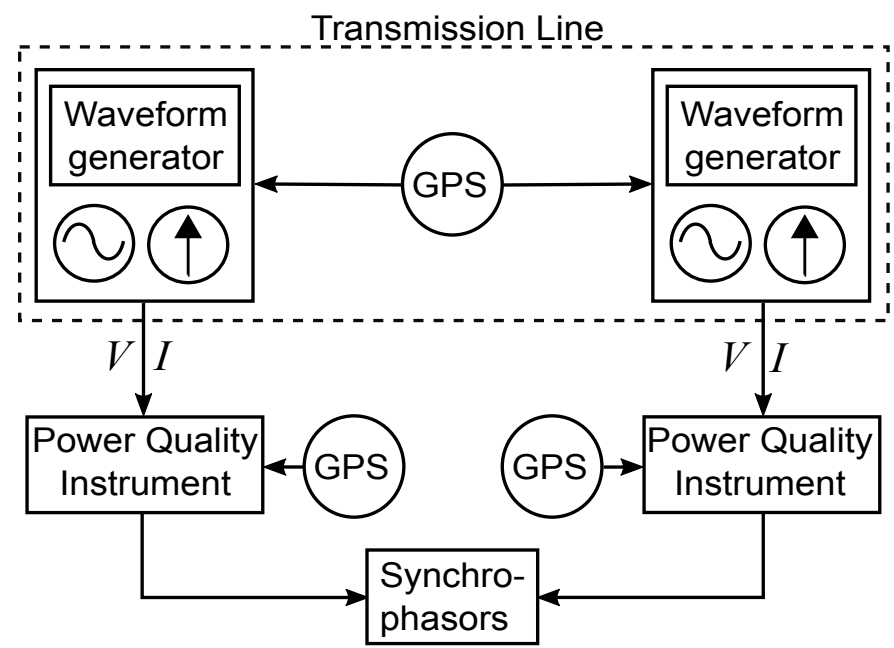

Fig. 4. Experimental setup for the laboratory-based synchrophasor measurements.

The waveform generators have a stated accuracy of $\pm 0.02 \%$ in magnitude and $\pm 0.005^{\circ}$ in phase angle, while the power quality instruments have a stated accuracy of $0.1 \%$ in magnitude; the power quality instruments do not have a stated accuracy for phase angle, however, during testing reported phase angles have been within $0.02^{\circ}$ of theoretical values. The GPS antennae provide UTC to within $1 \mu$ s to both the waveform generators and the power quality instruments.

By physically measuring the voltage and current signals, a realistic level of uncertainty due to the measurement units and time-tagging is introduced. Systematic errors in magnitudes and phase angles due to the remaining instrumentation channel
TABLE II

SYSTEMATIC ERRORS AND CORRECTIONS FOR ONE INDIVIDUAL CASE

\begin{tabular}{llllllll}
\hline & & $V_{s}$ & $V_{r}$ & $\mathrm{Net}^{1}$ & $I_{s}$ & $I_{r}$ & $\mathrm{Net}^{1}$ \\
\hline Magnitude & $\mathrm{SE}^{2}$ & 0.79 & 1.1 & -0.31 & -0.64 & 2.1 & -2.7 \\
$(\%)$ & $\mathrm{C}^{3}$ & -0.0058 & -0.29 & 0.28 & 5.6 & 2.7 & 2.9 \\
Phase angle & $\mathrm{SE}^{2}$ & -5.9 & 28 & -34 & 2.7 & 2.8 & -0.10 \\
$(\mathrm{mrad})$ & $\mathrm{C}^{3}$ & 12 & -22 & 34 & 6.7 & 6.0 & 0.70 \\
\hline
\end{tabular}

${ }^{1}$ Difference between sending and receiving end values

${ }^{2}$ Systematic error

${ }^{3}$ Corrections identified by the new method

were added to the reported phasors. Based on the accuracy classes of instrument transformers and characterization of instrumentation channels [?], [?], [?], errors in voltage and current magnitude were assumed to be up to $\pm 4 \%$ and $\pm 6 \%$, respectively, and errors in phase angle up to $\pm 0.04 \mathrm{rad}$ and $\pm 0.07 \mathrm{rad}$ for voltage and current, respectively. 100 cases of randomly selected systematic errors were applied to the phasor measurements and for each case, impedance parameters were estimated using both methods introduced in Sections II-B and II-A. For the existing method, a moving window spanning one hour of measurements was used in each estimation.

\section{Results}

First, one individual case of systematic errors is considered and then a summary of the results for 100 cases is given. Table II lists the systematic errors in magnitude and phase angle of voltage and current that have been applied in the individual case, together with the corrections that were identified by the proposed method. It can be recognized that the corrections for $V_{s}, V_{r}, I_{s}, I_{r}$ individually differ substantially from the systematic error; however, the 'net' correction, i.e. the difference between receiving and sending end values for each quantity matches the 'net' systematic errors very closely.

In Fig. 5 to 7 the estimated values of positive sequence resistance $R$, reactance $X$ and susceptance $B$ over time are shown. Nominal values as well as parameter estimates from both methods are included. All three graphs indicate close agreement between the nominal values and parameters estimated by the proposed method. In contrast, the existing method gives parameter estimates with greater and more variable deviation, especially for resistance. These observations are supported by the values in Table III, where it can be seen that the root-meansquare errors $E_{\Delta}$, as calculated in Appendix C, are below $5 \%$ for the new method for $R, X$ and $B$. Moreover, the standard deviation of error $\Sigma_{\Delta}$ is less than $1 \%$ for all three parameters computed by the proposed method, but it reaches $36 \%$ for the resistance values obtained by the existing method. Similarly, the resistance-based temperature estimates agree more closely with the conductor temperature for the new method.

Table IV shows the 50th, 75th and 95th percentiles of the root-mean-square (rms) and standard deviation of the errors in the parameters for both methods for 100 cases of systematic errors. $95 \%$ confidence intervals are given in square brackets next to the values; their width could be reduced by considering a larger number of cases, but in this instance, 100 cases give sufficiently narrow intervals to compare the two methods. 


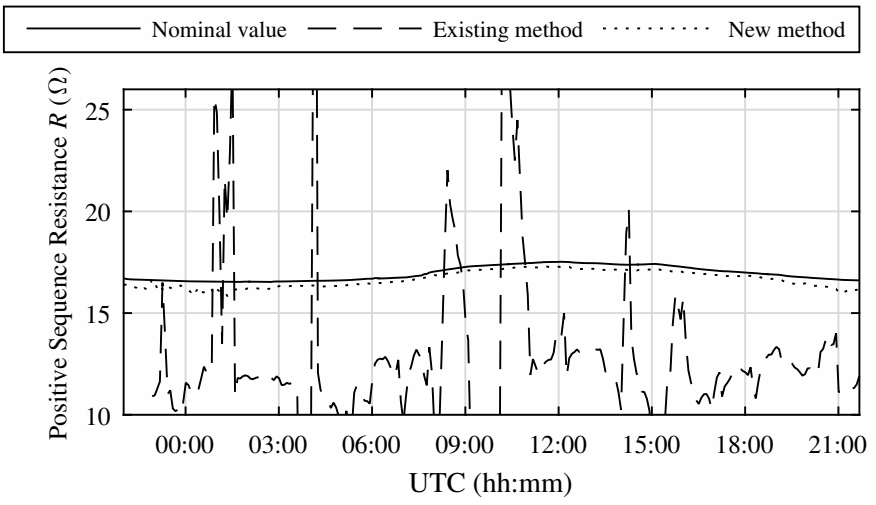

Fig. 5. Nominal and estimated values for positive sequence resistance $R$.

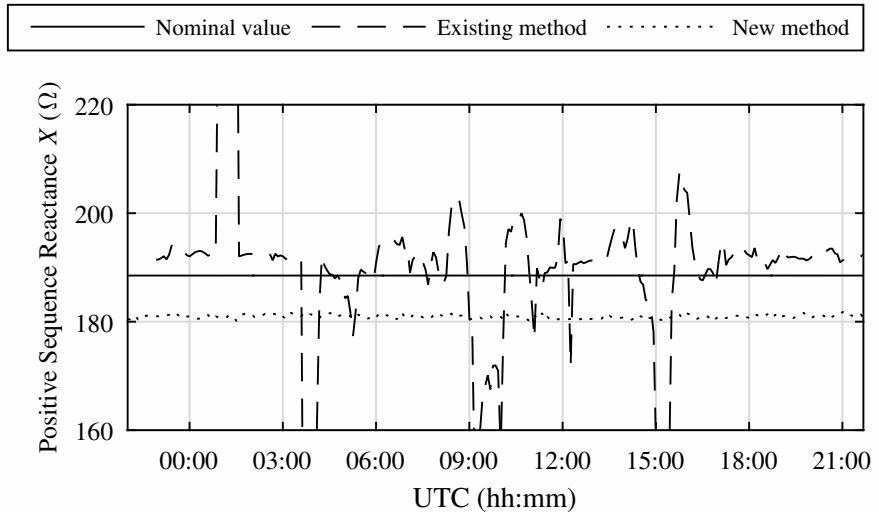

Fig. 6. Nominal and estimated values for positive sequence reactance $X$.

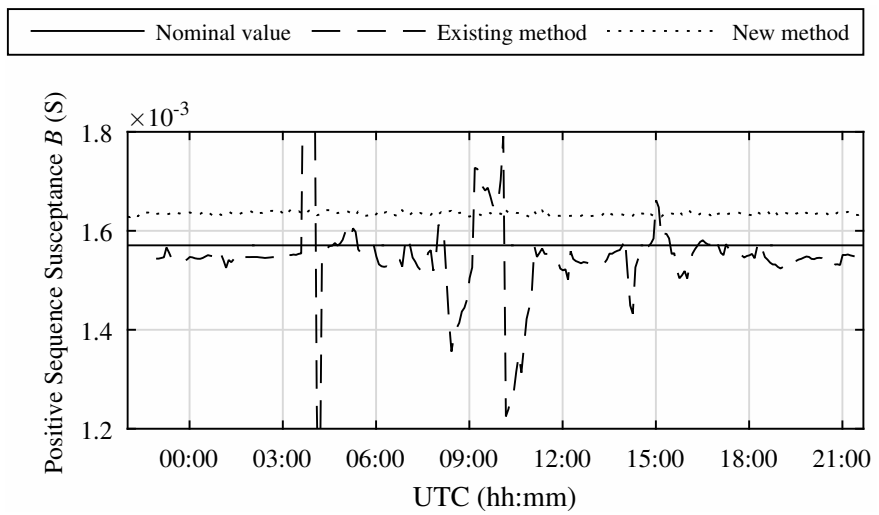

Fig. 7. Nominal and estimated values for positive sequence susceptance $B$.

The percentile values show that parameter errors are lower and less variable when estimated by the newly suggested method.

For instance, the 95th percentile of the rms error in $R$ is at $5.4 \%$ for the proposed method, while the existing method gives a value of $58 \%$. The standard deviation of the error is two orders of magnitude lower, at $0.67 \%$ compared to $30 \%$. In a similar manner, the new method gives lower values for the rms error and standard deviation of errors in reactance $X$ and susceptance $B$, as can be observed in Table IV. Across all 100 cases, rms error and standard deviation of errors in temperature estimates from the new method are approximately constant at $3.1^{\circ} \mathrm{C}$ and $1.7^{\circ} \mathrm{C}$, respectively.
TABLE III

PARAMETER ERRORS FOR ONE INDIVIDUAL CASE

\begin{tabular}{lllll}
\hline & & $R$ & $X$ & $B$ \\
\hline \multirow{2}{*}{$E_{\Delta}(\%)$} & $\mathrm{NM}^{1}$ & 1.8 & 4.0 & 4.1 \\
& $\mathrm{EM}^{2}$ & 36 & 10 & 6.7 \\
$\Sigma_{\Delta}(\%)$ & $\mathrm{NM}^{1}$ & 0.64 & 0.19 & 0.22 \\
& $\mathrm{EM}^{2}$ & 26 & 10 & 6.6 \\
\hline \multicolumn{5}{c}{} \\
& $\mathrm{NM}^{1}$ & 3.1 & 1.6 & \\
\hline & $\mathrm{EM}^{1}$ & 95 & 71 & \\
\hline
\end{tabular}

${ }^{1}$ New method

${ }^{2}$ Existing method

TABLE IV

ERRORS IN $R, X, B$ AND $T_{c}$ FOR 100 CASES

\begin{tabular}{cllll}
\hline & & \multicolumn{3}{c}{ Percentile } \\
& & \multicolumn{1}{c}{50 th } & \multicolumn{1}{c}{75 th } & \multicolumn{1}{c}{95 th } \\
\hline$E_{\Delta R}$ & $\mathrm{NM}^{1}$ & $2.5[1.7,2.9]$ & $3.5[3.2,4.2]$ & $5.4[4.9,6.0]$ \\
$(\%)$ & $\mathrm{EM}^{2}$ & $34[31,38]$ & $45[41,48]$ & $58[54,62]$ \\
$\Sigma_{\Delta R}$ & $\mathrm{NM}^{1}$ & $0.62[0.62,0.63]$ & $0.64[0.64,0.65]$ & $0.67[0.66,0.69]$ \\
$(\%)$ & $\mathrm{EM}^{2}$ & $26[26,27]$ & $28[27,28]$ & $30[29,31]$ \\
\hline$E_{\Delta X}$ & $\mathrm{NM}^{1}$ & $4.2[3.5,4.7]$ & $5.8[5.2,6.5]$ & $7.7[7.1,8.6]$ \\
$(\%)$ & $\mathrm{EM}^{2}$ & $10.1[10.1,10.2]$ & $10.4[10.3,10.6]$ & $11.0[10.8,11.3]$ \\
$\Sigma_{\Delta X}$ & $\mathrm{NM}^{1}$ & $0.19[0.19,0.19]$ & $0.19[0.19,0.19]$ & $0.20[0.19,0.20]$ \\
$(\%)$ & $\mathrm{EM}^{2}$ & $9.94[9.90,10.0]$ & $10.1[10.0,10.2]$ & $10.5[10.3,10.5]$ \\
\hline$E_{\Delta B}$ & $\mathrm{NM}^{1}$ & $4.4[3.7,4.9]$ & $6.1[5.5,6.8]$ & $8.4[7.7,9.3]$ \\
$(\%)$ & $\mathrm{EM}^{2}$ & $11[10,13]$ & $16[14,18]$ & $27[23,33]$ \\
$\Sigma_{\Delta B}$ & $\mathrm{NM}^{1}$ & $0.22[0.21,0.23]$ & $0.22[0.22,0.22]$ & $0.23[0.22,0.23]$ \\
$(\%)$ & $\mathrm{EM}^{2}$ & $7.0[6.6,7.5]$ & $8.0[7.7,8.4]$ & $9.3[8.6,10]$ \\
\hline$E_{\Delta T_{c}}$ & $\mathrm{NM}^{1}$ & $3.05[3.05,3.06]$ & $3.06[3.06,3.07]$ & $3.09[3.08,3.09]$ \\
$\left({ }^{\circ} \mathrm{C}\right)$ & $\mathrm{EM}^{2}$ & $90[84,101]$ & $119[111,129]$ & $159[146,170]$ \\
$\Sigma_{\Delta T_{c}}$ & $\mathrm{NM}^{1}$ & $1.64[1.63,1.65]$ & $1.66[1.65,1.68]$ & $1.71[1.70,1.71]$ \\
$\left({ }^{\circ} \mathrm{C}\right)$ & $\mathrm{EM}^{2}$ & $71[70,72]$ & $75[73,76]$ & $82[78,83]$ \\
\hline
\end{tabular}

${ }^{1}$ New method

${ }^{2}$ Existing method

The existing method on the other hand yields rms errors and standard deviations of above $70^{\circ} \mathrm{C}$.

\section{E. Analysis of results}

1) Comparison of methods: The results of the case study strongly suggest that the parameter identification method that has been proposed in this paper can be used to effectively calculate values of overhead line impedance and admittance that are consistent with expected physical behaviour. For a variety of cases of systematic errors, parameter estimates within $10 \%$ of known values were achieved. The new method's performance has been considerably better than that of an existing method for transmission line impedance parameter estimation. This difference in performance is attributed to various factors.

Firstly, in contrast to the existing method, the new method assumes that the synchrophasor measurements of voltage and current may contain systematic errors. As recommended by the authors of the existing method, bad data (individual measurements with large errors, for example due to instrumentation failure) may be removed before parameter estimation and synchronization errors corrected; but systematic errors will 
still be present in the remaining data set. Furthermore, the chi-square test may not detect all instances of bad data and synchronization errors, which means there is no removal or correction and thus no increase in impedance parameter accuracy.

Secondly, the existing method assumes constant parameters and thus attempts to estimate values from a limited time span of measurements in order to monitor thermal variations. If the set of measurements does not contain sufficient variation in operational states of the line, the problem becomes illconditioned, which means that the synchronization angle as well as impedance parameter estimates are likely to diverge significantly.

2) Estimated correction factors: Application of the proposed method to simulated measurements with known systematic errors has shown that the identified correction factors are not in good agreement with the size of the errors, yet a good level of impedance parameter accuracy is achieved. The reason is that calculation of impedance and admittance relies strongly on the differences of voltage and current between the two line ends; hence, correction of the 'net' errors across the line has a significant positive impact on impedance parameter calculation. On the other hand, it is difficult to detect and eliminate errors of the same sign and similar magnitudes at both line ends. But if the instrumentation channel at one line end is calibrated, they act as a reference and the estimated correction factors for the other line end become more accurate.

\section{DISCUSSION}

For practical applications in power networks, the proposed method could be utilized to determine correction factors and parameter estimates that can then be stored in databases of network management systems and thus support applications such as state estimation, protection, fault location, dynamic thermal line rating and safety monitoring. An initial measurement campaign for collecting synchrophasor and line temperature data would be necessary, but all or at least some of the instrumentation would not have to be installed permanently.

The proposed method has been used to identify positive sequence quantities at the fundamental frequency. Some applications require zero sequence or harmonic impedance of the transmission line, as well as corrections for the individual phase voltages and currents. An area for future work is the application of the proposed method to non-steady state conditions, which may provide measurements with significant unbalance, zero sequence or harmonic components.

In this paper, a general, simple error model based on constant transformer correction factors has been assumed. The actual systematic errors may behave in a different manner. One aspect of future work is to make the method adaptive, so that it can detect and compensate for different types of errors, with the aim of further improving parameter estimation accuracy.

\section{Conclusion}

The contribution of this paper is a novel method for synchrophasor-based overhead line impedance parameter estimation. The method differs from previous approaches in that it takes into account systematic errors in measured phasors and thermal variation of the line's electrical resistance. The results of a case study on laboratory measurements of an emulated long overhead transmission line suggest that the proposed method can determine impedance parameters effectively and more accurately than an existing method.

Estimation and monitoring of transmission line impedance parameters are essential building blocks in creating accurate power network models. Through continual improvement of the model accuracy, many functions of power network operation can be made more reliable and efficient, and new functions facilitated. These processes therefore play an important part in the development of smart grids, integration of renewable energy generation and in securing stable electricity provision.

\section{APPENDIX A}

\section{SELECTION OF MEASUREMENT SUBSETS}

Suppose there are $N \in \mathbb{N}$ available sets of synchrophasor measurements $V_{s_{i}}, I_{s_{i}}, V_{r_{i}}, I_{r_{i}}, i=1,2,3, \ldots, N$, then one can choose $\Gamma=1+(N-1)+(N-2)+\ldots+N=\sum_{m=0}^{N-1} N-m$ distinct subsets of consecutive measurements of sizes $n=1$ to $n=N$. Some subsets should be excluded because of their size and lack of variation of operational states. In order to ensure enough variation within the subsets, only those with a minimum range of current magnitudes are chosen. Given $\Gamma$ possible subsets, exclude those for which

$$
n<p ;|\max | I_{s_{i}}|-\min | I_{s_{i}}||<\frac{\eta}{n} \sum_{i=1}^{n}\left|I_{s_{i}}\right|,
$$

where $p \in \mathbb{N}$ is the minimum number of required measurement sets and $\eta \in \mathbb{R}_{\geq 0}$ is a factor that should be chosen at least one order of magnitude larger than the measurement uncertainties. For instance, if the measurement uncertainty in $I_{s}$ is less than $1 \%, \eta=10 \%$. Let the number of subsets that satisfy criterion (21) be $\Psi \in \mathbb{N}$, hence, the number of remaining subsets is $\Lambda=\Gamma-\Psi$.

\section{APPENDIX B \\ Calculation of $\hat{Z}_{\lambda_{i}}$ AND $\hat{Y}_{\lambda}$}

Given the set of correction factors $a_{s_{\lambda}}, a_{r_{\lambda}}, b_{s_{\lambda}}, b_{r_{\lambda}}$, values of $Z_{i}=R_{i}+j X_{i}$ and $Y_{i}=G_{i}+j B_{i}, i=1,2,3, \ldots, N$ are calculated using (1) and (2). Since $X, G, B$ are assumed constant, a single estimate can be obtained by taking the mean values $\bar{X}_{\lambda}=1 / N \sum_{i=1}^{N} X_{i}, \bar{G}_{\lambda}=1 / N \sum_{i=1}^{N} G_{i}, \bar{B}_{\lambda}=$ $1 / N \sum_{i=1}^{N} B_{i}$.

$R$ is not constant, but varies with temperature. The resistance-temperature relationship is assumed linear over the range of operating temperatures [?]:

$$
R_{i}=R_{0}\left(1+\alpha\left(T_{c_{i}}-T_{c_{0}}\right)\right)
$$

where $R_{0}, T_{c_{0}}, T_{c_{i}}, \alpha \in \mathbb{R} . T_{c_{i}}$ is the average conductor temperature at time instant $t_{i}, i=1,2,3, \ldots, N, R_{0}$ is the resistance value at a reference temperature $T_{c_{0}}$, and $\alpha$ is the resistance-temperature coefficient. In order to estimate values for constants $R_{0}$ and $\alpha$, (22) is expressed as

$$
R=x_{0}+x_{1} T_{c},
$$


where $x_{0}, x_{1} \in \mathbb{R}$ are unknown. The parameters $x_{0}, x_{1}$ of the resistance-temperature model are identified through linear least squares estimation from temperature measurements $T_{c_{i}}$ and calculated resistance values $R_{i}$. Define vectors $\mathbf{R}, \mathbf{T} \in \mathbb{R}^{N}, \mathbf{x} \in \mathbb{R}^{2}$ where $\mathbf{R}=\left[R_{1}, R_{2}, \ldots, R_{N}\right]^{T}, \mathbf{T}=$ $\left[T_{c_{1}}, T_{c_{2}}, \ldots, T_{c_{N}}\right]^{T}, \mathbf{x}=\left[x_{0}, x_{1}\right]$. The vectors are related by the $N$-dimensional matrix equation

$$
\mathbf{R}=\mathbf{T x}+\varepsilon,
$$

which is based on the theoretical model (23). $\varepsilon=\left[\varepsilon_{1}, \varepsilon_{2}, \ldots, \varepsilon_{N}\right]^{\mathrm{T}}$ models the deviation between synchrophasor-based values $\mathbf{R}$ and temperature-based values $\mathbf{T x}$. An estimate $\hat{\mathbf{x}}=\left[\hat{x}_{0}, \hat{x}_{1}\right]$ of $\mathbf{x}$ is computed by satisfying the least squares criterion, $\min \sum_{i=1}^{N} \varepsilon_{i}^{2}$ :

$$
\hat{\mathbf{x}}=\left(\mathbf{T}^{\mathrm{T}} \mathbf{T}\right)^{-1} \mathbf{T}^{\mathrm{T}} \mathbf{R} \text {. }
$$

Estimated values of reference resistance $\hat{R}_{0}$ and coefficient $\hat{\alpha}$ can be calculated from $\hat{x}_{0}$ and $\hat{x}_{1} \cdot \bar{X}_{\lambda}, \bar{G}_{\lambda}, \bar{B}_{\lambda}$ and temperature measurements $T_{c_{i}}$ are combined to give estimates of impedance and admittance, $\hat{Z}_{i_{\lambda}}, \hat{Y}_{\lambda}$ :

$$
\hat{Z}_{i_{\lambda}}=\hat{R}_{0}\left(1+\hat{\alpha}\left(T_{c_{i}}-T_{c_{0}}\right)\right)+j \bar{X}_{\lambda}, \hat{Y}_{\lambda}=\bar{G}_{\lambda}+j \bar{B}_{\lambda} .
$$

Conversely, temperature estimates $\hat{T}_{c_{i}}$ can be obtained from calculated resistance using the resistance-temperature model:

$$
\hat{T}_{c_{i}}=\left(R_{i}-\hat{x}_{0}\right) / \hat{x}_{1} \text {. }
$$

\section{APPENDIX C \\ METRICS FOR PARAMETER ACCURACY}

The first metric is the root-mean-square of the error $E_{\Delta P} \in$ $\mathbb{R}_{>0}$ gives an indication of how close $N$ parameter estimates $P_{i}$ are to a nominal value $P_{0}$. Define $\Delta P_{i}=P_{i}-P_{0}$ as the error in an individual parameter estimate. Then

$$
E_{\Delta P}=\frac{1}{P_{0}} \sqrt{\frac{1}{N} \sum_{i=1}^{N} \Delta P_{i}^{2}}
$$

The standard deviation of the error $\Sigma_{\Delta P} \in \mathbb{R}_{>0}$ reveals the variability of the errors in $P_{i}$.

$$
\Sigma_{\Delta P}=\frac{1}{P_{0}} \sqrt{\frac{1}{N} \sum_{i=1}^{N}\left(\Delta P_{i}-\mu_{\Delta P}\right)^{2}},
$$

where $\mu_{\Delta P}=1 / N \sum_{i=1}^{N} \Delta P_{i}$ is the mean parameter error.

\section{ACKNOWLEDGMENT}

The authors would like to thank Melvin Ferreira, Sane Rens, Hendrik Maree and Jan-Hendrik Hattingh of the North-West University as well as Tian van Rooyen, Charl Marais and Jacobus van Zyl of CT Lab for their invaluable assistance in conducting the laboratory-based measurements.
Deborah Ritzmann (S'15) was born in Germany. She received the B.Sc. degree in mathematics and physics from University College London, London, U.K., in 2012 and is currently pursuing the Ph.D. degree at the University of Reading, Reading, U.K., in collaboration with the National Physical Laboratory, Teddington, U.K..

The focus of her research is synchrophasor-based overhead line impedance measurement for dynamic line rating applications.

Johan Rens (M'03) received the M.Eng. and Ph.D. degrees in electrical engineering from the North-West University, Potchefstroom, S.A., in 1990 and 2002, respectively.

He has worked at the North-West University for the past 29 years and is currently a Professor with the School of Electrical and Electronic Engineering. $\mathrm{He}$ specializes in the quality of electrical energy, non-sinusoidal waveform conditions in modern power systems, the application of network coherent data/synchrophasors and power quality benchmarking.

Paul S. Wright received the B.Sc. and Ph.D. degrees in electrical and electronic engineering from the University of Surrey, Surrey, U.K., in 1987 and 2002, respectively.

He spent three years as a Research Fellow with the University of Surrey, where he was involved in the field of spacecraft sensors and attitude control. This was followed by three years with the Central Electricity Research Laboratory, where he was involved in advanced control systems. In 1992, he joined the National Physical Laboratory, Teddington, U.K., where he is currently a Principal Research Scientist specializing in ac measurements and waveform analysis.

William Holderbaum (M'01) received the Ph.D. degree in automatic control from the University of Lille, Lille, France, in 1999.

$\mathrm{He}$ was a Research Assistant with the University of Glasgow, Glasgow, U.K., from 1999 to 2001. He is currently a Professor with the University of Reading, Reading, U.K.. His current research interests include control theory and its applications.

Ben Potter (S'97-M'01) received the M.Eng. degree in engineering science from the University of Oxford, Oxford, U.K., in 2001, and the Ph.D. degree in modeling of induction machines from the University of Reading, Reading, U.K., in 2005.

He subsequently managed research and development activity for several years with Moog Components Group Ltd., Reading, including development work on wireless power transfer. He joined the University of Reading in 2009, where he is currently an Associate Professor of Energy Systems Engineering. His research interests include energy systems and power electronics with applications including energy storage, electric machines, wind turbines, and wireless power transfer. 